\title{
Long-term Outcomes of Undifferentiated-Type Early Gastric Cancer with Positive Horizontal Margins after Endoscopic Resection
}

\begin{abstract}
Hyo-Joon Yang ${ }^{1}$, Wan-Sik Lee ${ }^{2}$, Bong Eun Lee ${ }^{3}$, Ji Yong Ahn', Jae-Young Jang ${ }^{5}$, Joo Hyun Lim ${ }^{6}$, Su Youn Nam Jie-Hyun Kim ${ }^{8}$, Byung-Hoon Min ${ }^{9}$, Moon Kyung Joo ${ }^{10}$, Jae Myung Park ${ }^{11}$, Woon Geon Shin ${ }^{12}$, Hang Lak Lee ${ }^{13}$, Tae-Geun Gweon ${ }^{14}$, Moo In Park ${ }^{15}$, Jeongmin Choi ${ }^{16}$, Chung Hyun Tae ${ }^{17}$, Young-II Kim ${ }^{18}$, and II Ju Choi ${ }^{18}$

${ }^{1}$ Division of Gastroenterology, Department of Internal Medicine and Gastrointestinal Cancer Center, Kangbuk Samsung Hospital, Sungkyunkwan University School of Medicine, Seoul, ${ }^{2}$ Department of Internal Medicine, Chonnam National University Medical School, Gwangju, ${ }^{3}$ Department of Internal Medicine, Pusan National University School of Medicine, Busan, ${ }^{4}$ Division of Gastroenterology, Department of Internal Medicine, Asan Medical Center, University of Ulsan College of Medicine, ${ }^{5}$ Department of Internal Medicine, Kyung Hee University College of Medicine, ${ }^{6}$ Department of Internal Medicine, Healthcare Research Institute, Healthcare System Gangnam Center, Seoul National University Hospital, Seoul, ${ }^{7 D}$ ivision of Gastroenterology, Kyungpook National University Hospital and School of Medicine, Kyungpook National University, Daegu, ${ }^{8}$ Division of Gastroenterology, Department of Internal Medicine, Gangnam Severance Hospital, Yonsei University College of Medicine, ${ }^{9}$ Department of Medicine, Samsung Medical Center, Sungkyunkwan University School of Medicine, ${ }^{10}$ Department of Internal Medicine, Korea University Guro Hospital, Korea University College of Medicine, ${ }^{11}$ Department of Internal Medicine, Seoul St. Mary's Hospital, College of Medicine, The Catholic University of Korea, ${ }^{12}$ Department of Internal Medicine, Hallym University College of Medicine, ${ }^{13}$ Division of Gastroenterology, Department of Internal Medicine, Hanyang University College of Medicine, Seoul, ${ }^{14}$ Division of Gastroenterology, Department of Internal Medicine, Incheon St. Mary's Hospital, College of Medicine, The Catholic University of Korea, Incheon, ${ }^{15}$ Department of Internal Medicine, Kosin University College of Medicine, Busan, ${ }^{16}$ Department of Internal Medicine, Sanggye Paik Hospital, Inje University College of Medicine, ${ }^{17}$ Department of Internal Medicine, Ewha Womans University College of Medicine, Seoul, and ${ }^{18} \mathrm{Center}$ for Gastric Cancer, National Cancer Center, Goyang, Korea
\end{abstract}

\section{Article Info}

Received September 15, 2020

Revised November 18, 2020

Accepted December 1, 2020

Published online April 6, 2021

\section{Corresponding Author}

Wan-Sik Lee

ORCID https://orcid.org/0000-0002-8021-6228

E-mail jadelook@hanmail.net

Bong Eun Lee

ORCID https://orcid.org/0000-0003-2734-2134 E-mail bongsul@daum.net
Background/Aims: This study examined the long-term outcomes of undifferentiated-type early gastric cancer (UD EGC) with positive horizontal margins (HMs) after endoscopic resection (ER) and compared them between additional surgery and nonsurgical management.

Methods: From 2005 to 2015, a total of 1,124 patients with UD EGC underwent ER at 18 tertiary hospitals in Korea. Of them, 92 patients with positive HMs as the only noncurative factor $(n=25)$ or with both positive HMs and tumor size $>2 \mathrm{~cm}(\mathrm{n}=67)$ were included. These patients underwent additional surgery $(n=40)$, underwent additional endoscopic treatment $(n=6)$, or were followed up without further treatment $(n=46)$.

Results: No lymph node (LN) metastasis was found in patients who underwent additional surgery. During a median follow-up of 57.7 months (interquartile range, 27.6 to 68.8 months), no LN or distant metastases or gastric cancer-related deaths occurred in the overall cohort. At baseline, the residual cancer rate was 57.8\% (26/45) after additional surgery or ER. The 5-year local recurrence rate was $33.6 \%$ among patients who were followed up without additional treatment. The 5-year overall survival rates were $95.0 \%$ and $87.8 \%$ after additional surgery and nonsurgical management (endoscopic treatment or close follow-up), respectively (log-rank p=0.224). In the multivariate Cox regression analysis, nonsurgical management was not associated with an increased risk of mortality.

Conclusions: UD EGC with positive HMs after ER may have favorable long-term outcomes and a very low risk of $L N$ metastasis. Nonsurgical management may be suggested as an alternative, particularly for patients with old age or chronic illness. (Gut Liver 2021;15:723-731)

Key Words: Stomach neoplasms; Undifferentiated-type histology; Endoscopic mucosal resection; Margins of excision; Lymphatic metastasis 


\section{INTRODUCTION}

Endoscopic resection (ER) is accepted as a standard treatment for early gastric cancer (EGC) with a negligible risk of lymph node (LN) metastasis. ${ }^{1-4}$ When curative resection is achieved, ER provides excellent long-term outcomes compared with surgery. ${ }^{5}$ However, curative ER is not always possible, and additional gastrectomy with $\mathrm{LN}$ dissection is recommended when the resection is considered noncurative..$^{6-8}$ The guidelines also recommend nonsurgical management, such as additional endoscopic treatment or close observation without further treatment, as an alternative option for differentiated-type EGC in which a cancer-positive horizontal resection margin is the only noncurative factor, because of a very low risk of LN metastasis. ${ }^{2,9-11}$ In a previous study, no LN or distant metastasis or gastric cancer-related death occurred among 75 patients with differentiated-type EGC with a positive horizontal margin (HM) as the only noncurative factor during a median follow-up of 60 months. ${ }^{12}$ However, no such guidelines are available on the nonsurgical management for undifferentiated EGC with positive HM after ER. A recent study of 76 patients with positive HM only demonstrated that both differentiated and undifferentiated cancers can be managed with additional endoscopic treatments. ${ }^{13}$ However, in that study, the median follow-up duration after endoscopic treatments ranged from 13 to 21 months. Importantly, one patient with well-differentiated cancer who underwent argon plasma coagulation (APC) for positive HM developed peritoneal seeding after 4 years of follow-up. This can be a more crucial issue for an undifferentiated-type histology. Therefore, it is important to investigate the long-term outcomes, particularly the risk of LN or distant metastasis, of undifferentiated cancers with positive $\mathrm{HM}$ in order to evaluate the safety of nonsurgical management in these patients.

Therefore, this nationwide multicenter cohort study aimed to examine the long-term clinical outcomes of undifferentiated-type EGC (UD EGC) with positive HM after $\mathrm{ER}$, and to compare them between patients with additional surgery and those with nonsurgical management.

\section{MATERIALS AND METHODS}

\section{Patients}

In this retrospective cohort study, we reviewed the data of consecutive patients with UD EGC who underwent ER at 18 tertiary hospitals in six geographic areas in Korea between February 2005 and May 2015. We included patients with positive HM, which was defined as either en bloc resection with cancer cells identified in the horizontal resection margins of the resected specimen or a piecemeal resection. We excluded patients with positive vertical margin, submucosal invasion, lymphovascular invasion, or ulcer. Because we aimed to evaluate long-term outcomes, we further excluded patients with a history of gastric cancer, multiple gastric cancers, and initial follow-up loss. However, patients with tumor size $>2 \mathrm{~cm}$ were not excluded because it was reported that they had a low risk of LN metastasis. ${ }^{14,15}$ The baseline clinical, endoscopic, and histological data were retrospectively reviewed using the medical records. Chronic medical illness was defined as any of the following conditions: diabetes, cardiovascular disease, cerebrovascular disease, liver cirrhosis, or chronic kidney disease. One physician for each hospital was responsible for the data collection process. Two authors (H.J.Y. and Y.I.K.) independently reviewed, cleaned, and validated the overall data. The study protocol was reviewed and approved by the institutional review boards of all participating hospitals, and the requirement for informed consent was waived.

\section{Endoscopic resection}

Before ER, the lesion was identified with white-light endoscopy and chromoendoscopy using indigo carmine dye. Image-enhanced endoscopy was also used when available. Each patient underwent either endoscopic mucosal resection or endoscopic submucosal dissection (ESD). Several markings were made $5 \mathrm{~mm}$ outside the margin of the lesion, and epinephrine-containing saline solution was injected into the submucosal layer. Endoscopic mucosal resection was performed using a polypectomy snare. For ESD, after making a circumferential mucosal incision outside the markings, dissection along the submucosal plane was performed using a knife. Various knives, including IT-2 knife (Olympus, Tokyo, Japan), Flex knife (Olympus), or Dual knife (Olympus), were used according to the preference of the endoscopist.

\section{Histopathological assessment}

Endoscopically resected specimens were fixed in formalin and serially sectioned at 2-mm intervals to determine the presence of cancer cells in the horizontal resection margin. The histological types, tumor size, tumor invasion depth, presence of ulcer and lymphovascular invasion, and vertical resection margin were also evaluated microscopically. According to the Japanese guidelines, ${ }^{16}$ an undifferentiated-type histology was defined as poorly differentiated adenocarcinoma, signet ring cell carcinoma, or mucinous adenocarcinoma. 


\section{Additional treatment and follow-up}

The attending physician decided the additional treatment for positive HM from among additional surgery, additional ESD, APC, and close observation without further treatment, based on the tumor burden involved in the HM and the patient's general condition. The patients were divided into the surgery group, which included those who underwent additional surgical resection, and the nonsurgery group, which included those who were treated endoscopically or followed up without further treatment.

Upper endoscopy with a forceps biopsy at the ER site was conducted 3 months after the initial treatment, every 6 to 12 months for the first 3 years, and annually thereafter. Abdominal computed tomography was also performed every 6 to 12 months for the first 3 years, and then annually. ${ }^{2,17}$

Local recurrence was defined as a recurrent cancer occurring at the ER site. Synchronous or metachronous recurrence was defined as a new lesion detected at a lo- cation other than the previous ER site within or after 12 months from the initial ER, respectively. Overall survival was defined as the time from the initial ER to death from any cause. Survival status was determined from the medical records, and the cause of death was recorded as gastric cancer or other causes. The claims data of the Korean National Health Insurance Corporation were also accessed on March 2019. Disqualification of health insurance was considered to indicate death from an unknown cause, whereas maintenance of insurance was considered as censored on the date of screening. ${ }^{18}$

\section{Statistical analysis}

Continuous variables were compared using the Student t-test or the Mann-Whitney U test, and categorical variables were compared using the Pearson chi-square test, Fisher exact test, or linear-by-linear association test. Timedependent variables were evaluated using the Kaplan-Meier methods and log-rank test. The Cox regression analysis

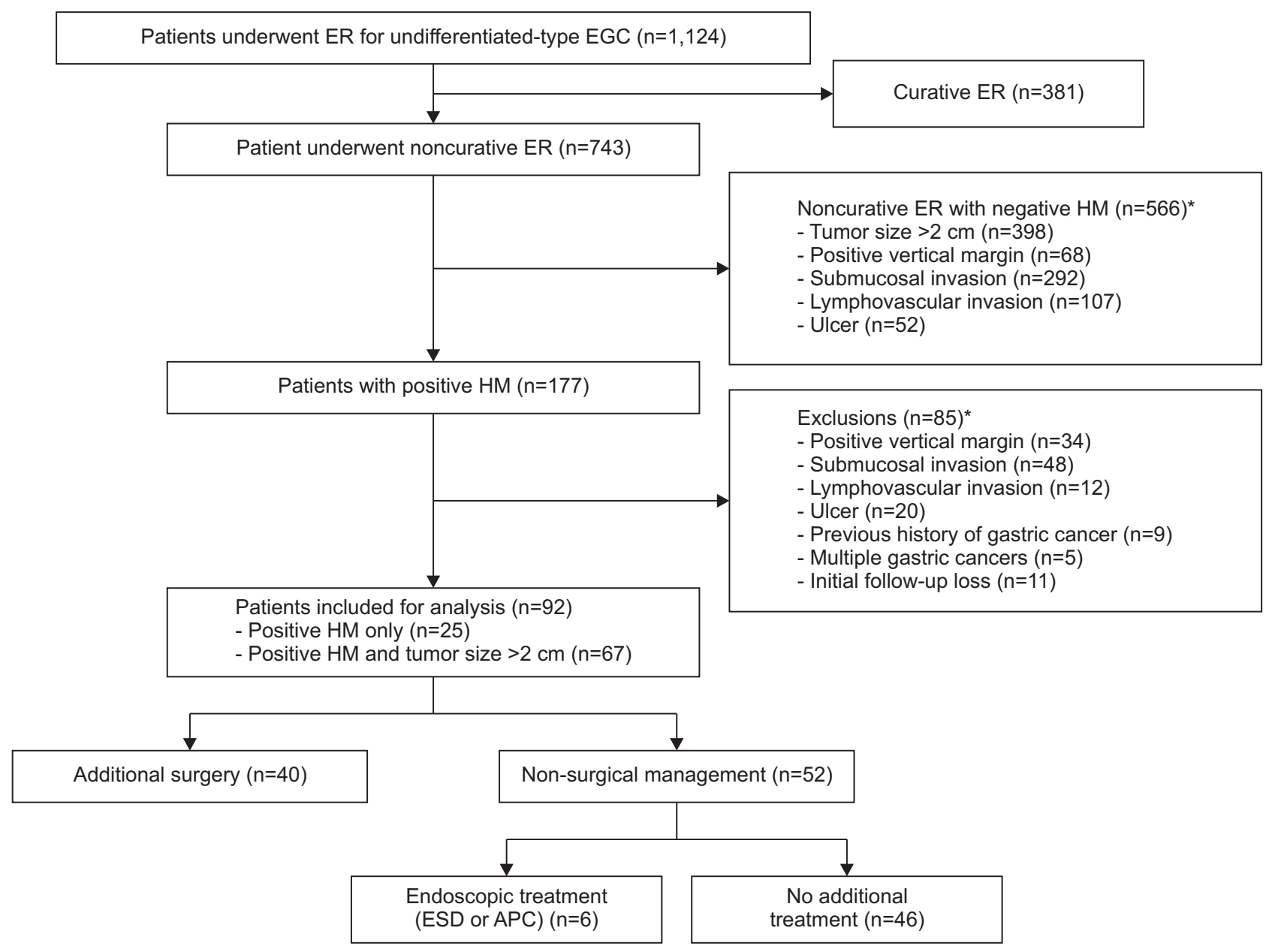

Fig. 1. Flowchart of the patients included in the analysis.

$E R$, endoscopic resection; EGC, early gastric cancer; HM, horizontal margin; ESD, endoscopic submucosal dissection; APC, argon plasma coagulation. *Some patients met more than one exclusion criteria. 
was also conducted for adjusted analyses of survival data. SPSS version 21.0 (SPSS Inc., Chicago, IL, USA) was used for the statistical analyses, and values of $\mathrm{p}<0.05$ were considered significant.

\section{RESULTS}

\section{Baseline characteristics of the included patients}

A total of 1,124 patients underwent ER for UD EGC, and 743 (66.0\%) underwent noncurative resection (Fig. 1). Of these patients, 177 were found to have a positive HM. Eighty-five patients were excluded for one or more of the following reasons: positive vertical margin $(n=34)$, submucosal invasion $(\mathrm{n}=48)$, lymphovascular invasion $(\mathrm{n}=12)$, ulcer $(n=20)$, previous history of gastric cancer $(n=9)$, multiple gastric cancers $(\mathrm{n}=5)$, and initial follow-up loss $(\mathrm{n}=11)$. Consequently, 92 patients with positive $\mathrm{HM}$ as the only noncurative factor $(n=25)$ or with both positive HM and tumor size $>2 \mathrm{~cm}(\mathrm{n}=67)$ were included in the analysis. Of them, 40 patients in the surgery group underwent additional surgery and 52 patients in the non-surgery group were treated with $\operatorname{ESD}(n=5)$ or APC $(n=1)$, or were followed up without additional treatment $(n=46)$.

Table 1 presents the baseline characteristics of the included patients. The surgery and non-surgery groups were significantly different in age, type of positive HM, and tumor size. The mean age \pm standard deviation was $57.6 \pm 9.1$ years in the surgery group and $65.0 \pm 13.8$ years in the nonsurgery group $(\mathrm{p}=0.003)$. No patient with piecemeal resection (0/14) and only $20.0 \%(5 / 25)$ of the patients with tumor size $\leq 2 \mathrm{~cm}$ underwent additional surgery. Meanwhile, patients with positive HM after en bloc resection or with larger tumor size were more likely to be treated with additional surgery (both $\mathrm{p}<0.05)$.

Table 1. Baseline Characteristics of the Included Patients

\begin{tabular}{|c|c|c|c|}
\hline Characteristics & Surgery $(n=40)$ & Non-surgery ( $n=52)$ & p-value \\
\hline Age, yr & $57.6 \pm 9.1$ & $65.0 \pm 13.8$ & 0.003 \\
\hline Male sex & $21(52.5)$ & 32 (61.5) & 0.384 \\
\hline ASA class & & & 0.813 \\
\hline I & $22(55.0)$ & 32 (61.5) & \\
\hline ॥ & $18(45.0)$ & $18(34.6)$ & \\
\hline III-IV & 0 & $2(3.8)$ & \\
\hline Current or past smoking & $11(27.5)$ & 14 (26.9) & 0.951 \\
\hline Chronic medical illness* & $7(17.5)$ & 9 (17.3) & 0.985 \\
\hline Helicobacter pylori infection & $21 / 31(67.7)$ & $31 / 48(64.6)$ & 0.773 \\
\hline Location of tumor & & & 0.695 \\
\hline Upper/middle & $38(95.0)$ & $47(90.4)$ & \\
\hline Lower & $2(5.0)$ & $5(9.6)$ & \\
\hline Macroscopic appearance & & & 0.240 \\
\hline Elevated & $11(27.5)$ & 9 (17.3) & \\
\hline Depressed/flat & 29 (72.5) & 43 (82.7) & \\
\hline Histology & & & 0.546 \\
\hline PDA & $21(52.5)$ & $24(46.2)$ & \\
\hline $\mathrm{SRC}$ & 19 (47.5) & 28 (53.8) & \\
\hline Resection method & & & 0.649 \\
\hline EMR & $3(7.5)$ & $2(3.8)$ & \\
\hline ESD & 37 (92.5) & 50 (96.2) & \\
\hline Type of positive HM & & & $<0.001$ \\
\hline En bloc with positive HM & $40(100)$ & $38(73.1)$ & \\
\hline Piecemeal resection & 0 & $14(26.9)$ & \\
\hline Tumor size, $\mathrm{cm}$ & $3.4(1.2-6.2)$ & $2.8(0.6-7.0)$ & 0.036 \\
\hline Tumor size, $\mathrm{cm}$ & & & 0.012 \\
\hline$\leq 2$ & 5 (12.5) & 20 (38.5) & \\
\hline $2-4$ & $23(57.5)$ & $17(32.7)$ & \\
\hline$>4$ & $12(30.0)$ & 15 (28.8) & \\
\hline
\end{tabular}

Data are presented as mean $\pm S D$, number (\%), or median (range).

ASA, American Society of Anesthesiologists; PDA, poorly differentiated adenocarcinoma; SRC, signet ring cell carcinoma; EMR, endoscopic mucosal resection; ESD, endoscopic submucosal dissection; HM, horizontal margin.

${ }^{*}$ Chronic medical illnesses included diabetes, cardiovascular disease, cerebrovascular disease, liver cirrhosis, and chronic kidney disease. 


\section{Risk of residual cancer and recurrence after positive HM}

Among the 40 patients in the surgery group, LN metastasis was not noted in the surgical specimen of any patient (Table 2). However, residual cancer was detected in 65.0\% $(26 / 40)$ of the patients who underwent additional surgery and in $0.0 \%(0 / 5)$ of those who underwent additional ESD. The histological outcome of residual cancer was not available in one patient who underwent additional APC because the patient lost to follow-up after APC.

The overall follow-up duration was 57.7 months (interquartile range, 27.6 to 68.8 months) for recurrence and gastric cancer-related mortality. During the followup, no regional LN or distant metastasis was detected in both groups. Further, no local, synchronous, or metachronous recurrence was found in the surgery group. On the contrary, 11 (21.2\%) local recurrences and four (7.7\%) synchronous or metachronous recurrences were recorded in the non-surgery group. Specifically, local recurrence occurred only in the 46 patients who were followed up without additional treatment. The cumulative risk of local recurrence was $13.8 \%, 20.2 \%$, and $33.6 \%$ after 1,3 , and 5 years of follow-up, respectively (Fig. 2A). With respect to tumor size, the 5-year risk of local recurrence was $17.7 \%$ for tumor size $\leq 2 \mathrm{~cm}$ and $43.4 \%$ for tumor size $>2 \mathrm{~cm}$ (logrank $\mathrm{p}=0.051$ ). The clinical course after local recurrence is presented in Fig. 2B. Patients with local recurrence were treated with surgery $(n=4), \operatorname{ESD}(n=5)$, or $\operatorname{APC}(n=2)$. The

Table 2. Summary of Clinical Outcomes

\begin{tabular}{|c|c|c|c|}
\hline Outcome & Surgery $(n=40)$ & Non-surgery (n=52) & $\mathrm{p}$-value \\
\hline \multicolumn{4}{|l|}{ Outcome of additional treatment } \\
\hline Residual cancer & $26(65.0)$ & $0 / 5(0) *$ & - \\
\hline LN metastasis & 0 & - & - \\
\hline \multicolumn{4}{|l|}{ Recurrence } \\
\hline Follow-up duration, mo & $58.9(37.9-68.4)$ & $48.7(15.1-69.3)$ & 0.228 \\
\hline Local recurrence & 0 & $11(21.2)$ & 0.002 \\
\hline Regional LN or distant metastasis & 0 & 0 & NA \\
\hline Synchronous or metachronous recurrence ${ }^{+}$ & 0 & 4 (7.7) & 0.130 \\
\hline Mortality & $1(2.5)$ & $16(9.1)$ & 0.208 \\
\hline Follow-up duration, mo & $88.5(67.4-112.0)$ & $79.2(60.0-97.3)$ & 0.337 \\
\hline Gastric cancer & 0 & 0 & \\
\hline Other cause & 1 (33.3) & $3(37.5)$ & \\
\hline Unknown cause & $2(66.7)$ & $5(62.5)$ & \\
\hline
\end{tabular}

Data are presented as number (\%) or median (interquartile range).

LN, lymph node; NA, not available.

*Data were available for patients who underwent additional endoscopic submucosal dissection; ${ }^{\dagger}$ Synchronous recurrence and metachronous recurrence were defined as the recurrence of a new lesion detected at a previously uninvolved site during follow-up $\leq 1$ year and $>1$ year after initial treatment, respectively.

A

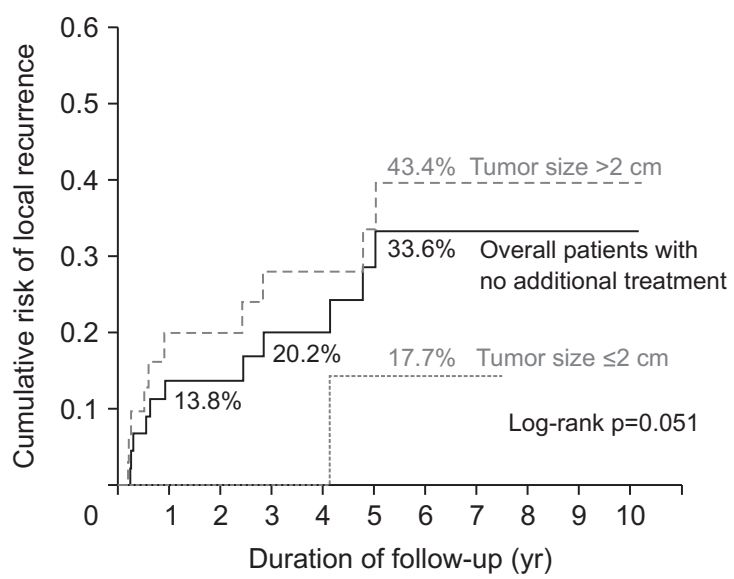

B

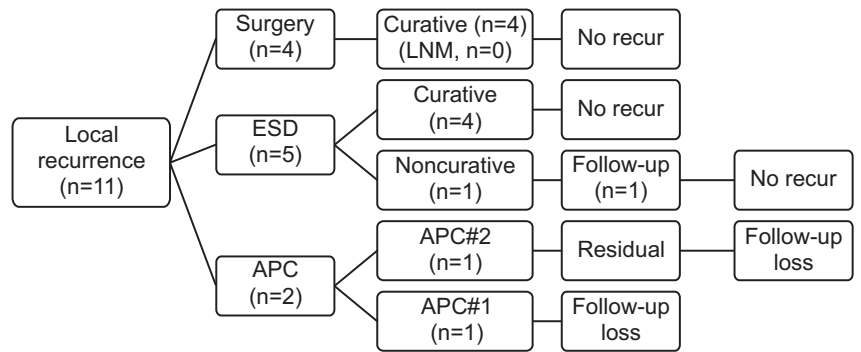

No. at risk $4 \begin{array}{llllll}30 & 30 & 10 & 4 & 1\end{array}$

Fig. 2. (A) Cumulative risk of local recurrence in patients who were followed up without additional treatment. (B) Clinical course of patients who developed local recurrence during the follow-up period. ESD, endoscopic submucosal dissection; LNM, lymph node metastasis; APC, argon plasma coagulation. 


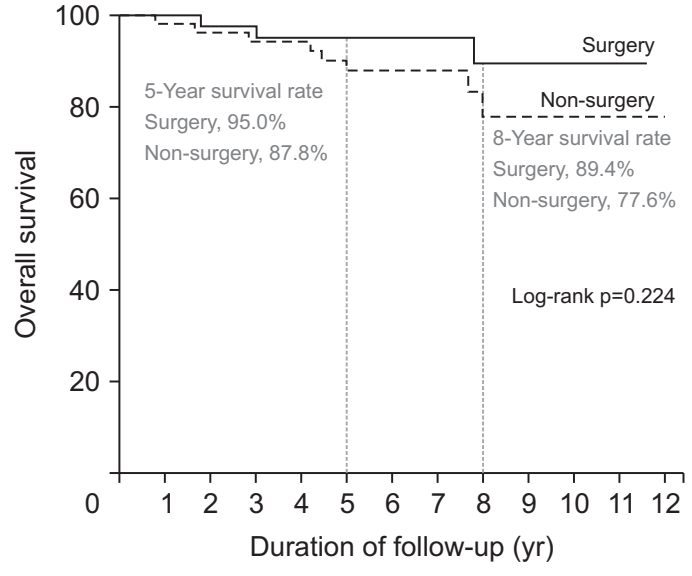

No. at risk

$\begin{array}{llllllll}\text { Surgery } & 40 & 39 & 37 & 28 & 16 & 5 & 0\end{array}$

$\begin{array}{llllllll}\text { Non-surgery } & 52 & 50 & 48 & 32 & 14 & 8 & 0\end{array}$

Fig. 3. Overall survival according to the management strategy of patients with positive horizontal margins after endoscopic resection. patients did not develop further recurrence after surgery or ESD during the remaining follow-up period. However, in the two patients who treated with APC, one patient still had residual cancer after APC and another patient was not assessed for treatment outcome because of follow-up loss. Four patients with synchronous or metachronous recurrences also did not develop further recurrence after curative $(n=3)$ or noncurative $\operatorname{ESD}(n=1)$.

\section{Survival outcomes according to management for positive HM}

During a median follow-up of 81.3 months (interquartile range, 62.7 to 106.7 months) for overall survival, no gastric cancer-related death was recorded, although two and five patients in the surgery and non-surgery groups died of an unknown cause, respectively (Table 2). The 5- and 8-year overall survival rates were $95.0 \%$ and $89.4 \%$, respectively, in the surgery group and $87.8 \%$ and $77.6 \%$, respectively, in the non-surgery group ( $\mathrm{p}=0.224)$ (Fig. 3). In multivariate Cox regression analysis, age (hazard ratio [HR], 1.19; 95\% confidence interval [CI], 1.07 to $1.34 ; \mathrm{p}=0.002)$, smoking

Table 3. Univariate and Multivariable Analyses for Mortality

\begin{tabular}{|c|c|c|c|c|}
\hline \multirow{2}{*}{ Covariate } & \multicolumn{2}{|c|}{ Univariate analysis } & \multicolumn{2}{|c|}{ Multivariable analysis } \\
\hline & $\operatorname{HR}(95 \% \mathrm{Cl})$ & $\mathrm{p}$-value & $\operatorname{HR}(95 \% \mathrm{Cl})$ & $p$-value \\
\hline Age, yr & $1.11(1.04-1.17)$ & 0.001 & $1.19(1.07-1.34)$ & 0.002 \\
\hline Sex & & 0.112 & & \\
\hline Female & 1 & & & \\
\hline Male & $3.47(0.75-16.06)$ & & & \\
\hline Smoking & & 0.028 & & 0.006 \\
\hline Never & 1 & & 1 & \\
\hline Current or past & $2.80(1.16-12.48)$ & & $8.45(1.82-39.18)$ & \\
\hline Chronic medical illness* & & 0.019 & & 0.011 \\
\hline No & 1 & & 1 & \\
\hline Yes & $4.93(1.30-18.78)$ & & $7.27(1.59-33.30)$ & \\
\hline ASA class & & 0.275 & & \\
\hline I & 1 & & & \\
\hline II-IV & $1.94(0.59-6.38)$ & & & \\
\hline Helicobacter pylori & & 0.168 & & \\
\hline Absent & 1 & & & \\
\hline Present & $0.43(0.13-4.42)$ & & & \\
\hline Histology & & 0.340 & & \\
\hline $\mathrm{SRC}$ & 1 & & & \\
\hline Non-SRC & $1.82(0.53-6.22)$ & & & \\
\hline Type of positive HM & & 0.851 & & \\
\hline Piecemeal resection & 1 & & & \\
\hline En bloc with positive HM & $0.86(0.19-4.01)$ & & & \\
\hline Tumor size, cm & & 0.867 & & \\
\hline$\leq 2$ & 1 & & & \\
\hline$>2$ & $0.89(0.24-3.37)$ & & & \\
\hline Additional treatment & & 0.236 & & 0.401 \\
\hline Surgery & 1 & & 1 & \\
\hline Non-surgery & $2.23(0.59-8.43)$ & & $0.49(0.09-2.61)$ & \\
\hline
\end{tabular}

$\mathrm{HR}$, hazard ratio; $\mathrm{Cl}$, confidence interval; ASA, American Society of Anesthesiologists; SRC, signet ring cell carcinoma; HM, horizontal margin. ${ }^{*}$ Chronic medical illnesses included diabetes, cardiovascular disease, cerebrovascular disease, liver cirrhosis, and chronic kidney disease. 
(HR, 8.45; 95\% CI, 1.82 to $39.18 ; \mathrm{p}=0.006$ ), and chronic medical illness (HR, 7.27; 95\% CI, 1.59 to 33.30; $\mathrm{p}=0.011$ ) were independently associated with mortality (Table 3 ). After adjustment for these variables, nonsurgical management was not associated with an increased risk of mortality (HR, 0.49; 95\% CI, 0.09 to $2.61 ; \mathrm{p}=0.401$ ).

\section{DISCUSSION}

In the present study, we included 92 patients with UD EGC who had positive HM after ER. To our knowledge, this is the largest sample size from a nationwide multicenter cohort in Korea. We found no case of LN metastasis after additional surgery, and no LN or distant metastasis or gastric cancer-related death occurred in the overall cohort during a median follow-up of 58 months. In addition, nonsurgical management was not associated with an increased risk of overall mortality during a median followup of 81 months. Our findings suggest that the risk of LN metastasis may be very low in UD EGC with positive HM, and that nonsurgical management can be an alternative for these cases.

Many studies have evaluated various aspects of EGC with positive HM after ER, including predictors of positive $\mathrm{HM},{ }^{19}$ outcomes of additional endoscopic treatment, ${ }^{9-11}$ risk factors for local recurrence, ${ }^{12,20,21}$ and long-term outcomes. ${ }^{12}$ However, no or only a small number of cases of undifferentiated-type cancer were included in those studies. A recent study reported data on 76 patients with positive $\mathrm{HM}$ after ER, including 26 patients with UD EGC. ${ }^{13}$ In that study, no LN or distant metastasis was detected among patients with UD EGC. However, the study was also limited in that the follow-up duration was relatively short and patients undergoing close observation without additive treatment were not included. In our study, we included 92 patients with UD EGC with positive HM after ER and followed them up for a median of 5 years. Our study demonstrates that these patients may have a very low risk of LN metastasis, with higher credibility than previous smallsized studies. To obtain a large sample size, we also included patients with tumor size $>2 \mathrm{~cm}$. Although tumor size $>2$ $\mathrm{cm}$ is associated with LN metastasis in undifferentiatedtype cancer, ${ }^{3}$ a more recent study suggested that the risk of LN metastasis would be low in patients with a tumor size of $>2 \mathrm{~cm}$ as the only risk factor than in those with other risk factors. ${ }^{14}$ Therefore, our results suggest that a positive HM status with an undifferentiated-type histology after ER may not increase the risk of LN metastasis if there are no further risk factors.

In our study, the residual cancer rate was 58\% (26/45) among patients who underwent additional surgery or ESD. Our results are consistent with those of previous studies that reported a $50 \%$ to $86 \%$ rate of residual cancer. ${ }^{9,13,19}$ The rate of local recurrence was $33.6 \%$ after 5 years of followup among patients who were followed up without additional treatment in our study. Although the rate was lower in patients with tumor size $\leq 2 \mathrm{~cm}(18 \%)$ than in those with tumor size $>2 \mathrm{~cm}(43 \%)$, these findings are also consistent with previous results demonstrating a rate of $13 \%$ to $36 \%{ }^{9,12,13,20}$ Thus, our results suggest that the risk of residual cancer or local recurrence due to positive HM after ER would be similar between the undifferentiated-type histology that was observed in our study and the differentiatedtype histology that was reported in previous studies.

The overall survival rates in the non-surgery group were only $88 \%$ and $78 \%$ after 5 and 8 years of follow-up. These seemed lower than those in the surgery group, which were $95 \%$ and $89 \%$ after 5 and 8 years of follow-up, respectively. However, these may not be due to gastric cancer because no metastatic disease and gastric cancer-related death occurred during 5 years of follow-up. This was supported by the results of the Cox regression analysis in which age, smoking, and chronic medical illness were independent predictors of overall survival. In fact, patients in the nonsurgery group were significantly older than those in the surgery group at baseline. Therefore, these results suggest that nonsurgical management could be recommended for patients with old age or chronic illness.

ESD was previously suggested to be an effective treatment for residual or locally recurrent cancer with positive HM after ER. ${ }^{10,11,13,19}$ In our study, 10 patients were treated with ESD for residual cancer or local recurrence and did not experience further recurrence after ESD. On the contrary, only three patients were treated with APC. One patient underwent additional APC for positive HM before any local recurrence was detected, and the other two underwent APC after local recurrence was diagnosed. Because two patients were lost to follow-up after APC, the treatment outcome was available in only one patient in whom APC failed to eradicate recurrent cancer. Therefore, our results support the use of ESD as additive treatment for residual cancer or salvage treatment for local recurrence. However, the data from our study were not sufficient to evaluate the effectiveness of APC.

The strength of our study lies in the large sample size and long-term follow-up duration, reliably suggesting that a positive HM with undifferentiated-type histology after ER has a very low risk of LN metastasis. However, the limitation of our study was that UD EGCs $>2 \mathrm{~cm}$ in diameter were included in addition to EGCs $\leq 2 \mathrm{~cm}$. Our data should not be interpreted to mean that UD EGCs $>2 \mathrm{~cm}$ in 
diameter may be subject to ER because we did not include all patients with tumor size $>2 \mathrm{~cm}$. Another limitation is the retrospective design based on a medical record review and the lack of pathological slide review. Consequently, we could not measure the length of positive HM that was suggested as an important risk factor in the selection of additive treatment modality or for the prediction of local recurrence. ${ }^{12,13,20,21}$ However, it may be unlikely that the length of positive HM influences the long-term risk of LN or distant metastasis, which was the focus of the present study.

In conclusion, UD EGC with positive HM after ER may have favorable long-term outcomes and a very low risk of LN metastasis. Nonsurgical management such as additional endoscopic treatment or close follow-up without additional treatment may be suggested as an alternative for patients with old age or chronic illness.

\section{CONFLICTS OF INTEREST}

J.Y.A. is an editorial board member of the journal but was not involved in the peer reviewer selection, evaluation, or decision process of this article. No other potential conflicts of interest relevant to this article were reported.

\section{ACKNOWLEDGEMENTS}

This work was supported by the Korean College of Helicobacter and Upper Gastrointestinal Research (Woon Geon Shin in 2017 and Hyo-Joon Yang in 2018) and the National Research Foundation of Korea (2020R1G1A1010927).

\section{AUTHOR CONTRIBUTIONS}

Study concept and design: H.J.Y., J.Y.A, J.H.K., W.G.S., I.J.C. Data acquisition: all authors. Data analysis and interpretation: H.J.Y. Drafting of the manuscript: H.J.Y. Critical revision of the manuscript for important intellectual content: all authors. Statistical analysis: H.J.Y. Obtained funding: H.J.Y., W.G.S. Administrative, technical, or material support: J.Y.A., J.H.K., W.G.S., Y.I.K., I.J.C. Study supervision: W.S.L., B.E.L.

\section{ORCID}

Hyo-Joon Yang https://orcid.org/0000-0002-0265-672X Wan-Sik Lee https://orcid.org/0000-0002-8021-6228

Bong Eun Lee https://orcid.org/0000-0003-2734-2134
Ji Yong Ahn

https://orcid.org/0000-0002-0030-3744 Jae-Young Jang Joo Hyun Lim https://orcid.org/0000-0002-7930-1468 https://orcid.org/0000-0002-8437-096X https://orcid.org/0000-0002-5568-7714 Su Youn Nam Jie-Hyun Kim https://orcid.org/0000-0002-9198-3326
Byung-Hoon Min https://orcid.org/0000-0001-8048-361X Moon Kyung Joo https://orcid.org/0000-0001-6050-3695 Jae Myung Park https://orcid.org/0000-0002-1534-7467 Woon Geon Shin https://orcid.org/0000-0002-9851-5576 Hang Lak Lee https://orcid.org/0000-0002-2825-3216 Tae-Geun Gweon https://orcid.org/0000-0002-0884-7228 Moo In Park https://orcid.org/0000-0003-2071-6957 Jeongmin Choi https://orcid.org/0000-0001-6976-9887 Chung Hyun Tae https://orcid.org/0000-0002-0764-7793 Young-Il Kim https://orcid.org/0000-0002-2614-3419 Il Ju Choi

\section{REFERENCES}

1. Guideline Committee of the Korean Gastric Cancer Association (KGCA), Development Working Group \& Review Panel. Korean practice guideline for gastric cancer 2018: an evidence-based, multi-disciplinary approach. J Gastric Cancer 2019;19:1-48.

2. Japanese Gastric Cancer Association. Japanese gastric cancer treatment guidelines 2018 (5th edition). Gastric Cancer 2021;24:1-21.

3. Hirasawa T, Gotoda T, Miyata S, et al. Incidence of lymph node metastasis and the feasibility of endoscopic resection for undifferentiated-type early gastric cancer. Gastric Cancer 2009;12:148-152.

4. Gotoda T, Yanagisawa A, Sasako M, et al. Incidence of lymph node metastasis from early gastric cancer: estimation with a large number of cases at two large centers. Gastric Cancer 2000;3:219-225.

5. Lee S, Choi KD, Han M, et al. Long-term outcomes of endoscopic submucosal dissection versus surgery in early gastric cancer meeting expanded indication including undifferentiated-type tumors: a criteria-based analysis. Gastric Cancer 2018;21:490-499.

6. Suzuki H, Oda I, Abe S, et al. Clinical outcomes of early gastric cancer patients after noncurative endoscopic submucosal dissection in a large consecutive patient series. Gastric Cancer 2017;20:679-689.

7. Hatta W, Gotoda T, Oyama T, et al. Is radical surgery necessary in all patients who do not meet the curative criteria for endoscopic submucosal dissection in early gastric cancer? A multi-center retrospective study in Japan. J Gastroenterol 2017;52:175-184

8. Yang HJ, Kim SG, Lim JH, et al. Predictors of lymph node 
metastasis in patients with non-curative endoscopic resection of early gastric cancer. Surg Endosc 2015;29:1145-1155.

9. Han JP, Hong SJ, Choi MH, et al. Clinical outcomes of early gastric cancer with lateral margin positivity after endoscopic submucosal dissection. Gastrointest Endosc 2013;78:956961.

10. Bae SY, Jang TH, Min BH, et al. Early additional endoscopic submucosal dissection in patients with positive lateral resection margins after initial endoscopic submucosal dissection for early gastric cancer. Gastrointest Endosc 2012;75:432436.

11. Sekiguchi M, Suzuki H, Oda I, et al. Favorable long-term outcomes of endoscopic submucosal dissection for locally recurrent early gastric cancer after endoscopic resection. Endoscopy 2013;45:708-713.

12. Sekiguchi M, Suzuki H, Oda I, et al. Risk of recurrent gastric cancer after endoscopic resection with a positive lateral margin. Endoscopy 2014;46:273-278.

13. Kim HW, Kim JH, Park JC, et al. Additive endoscopic resection may be sufficient for patients with a positive lateral margin after endoscopic resection of early gastric cancer. Gastrointest Endosc 2017;86:849-856.

14. Horiuchi Y, Ida S, Yamamoto N, et al. Feasibility of further expansion of the indications for endoscopic submucosal dissection in undifferentiated-type early gastric cancer. Gastric Cancer 2020;23:285-292.
15. Yang HJ, Nam SY, Min BH, et al. Clinical outcomes of endoscopic resection for undifferentiated intramucosal early gastric cancer larger than $2 \mathrm{~cm}$. Gastric Cancer 2021;24:435444.

16. Japanese Gastric Cancer Association. Japanese classification of gastric carcinoma: 3rd English edition. Gastric Cancer 2011;14:101-112.

17. Lee JH, Kim JG, Jung HK, et al. Clinical practice guidelines for gastric cancer in Korea: an evidence-based approach. J Gastric Cancer 2014;14:87-104.

18. Kim JH, Kim K, Park SJ, et al. Comparative effectiveness of abdominal versus laparoscopic radical hysterectomy for cervical cancer in the postdissemination era. Cancer Res Treat 2019;51:788-796.

19. Lee JH, Lee JH, Kim KM, Kang KJ, Min BH, Kim JJ. Clinicopathological factors of multiple lateral margin involvement after endoscopic submucosal dissection for early gastric cancer. Surg Endosc 2015;29:3460-3468.

20. Kim TK, Kim GH, Park DY, et al. Risk factors for local recurrence in patients with positive lateral resection margins after endoscopic submucosal dissection for early gastric cancer. Surg Endosc 2015;29:2891-2898.

21. Yoon H, Kim SG, Choi J, et al. Risk factors of residual or recurrent tumor in patients with a tumor-positive resection margin after endoscopic resection of early gastric cancer. Surg Endosc 2013;27:1561-1568. 